Article

\title{
On the Convolution Quadrature Rule for Integral Transforms with Oscillatory Bessel Kernels
}

\author{
Junjie Ma 1,2,* and Huilan Liu 1,2,* \\ 1 School of Mathematics and Statistics, Guizhou University, Guiyang 550025, China \\ 2 Guizhou Provincial Key Laboratory of Public Big Data, Guizhou University, \\ Guiyang 550025, China \\ * Correspondence: jjma@gzu.edu.cn (J.M.); hlliu5@gzu.edu.cn (H.L.)
}

Received: 13 June 2018; Accepted: 23 June 2018; Published: 25 June 2018

check for updates

\begin{abstract}
Lubich's convolution quadrature rule provides efficient approximations to integrals with special kernels. Particularly, when it is applied to computing highly oscillatory integrals, numerical tests show it does not suffer from fast oscillation. This paper is devoted to studying the convergence property of the convolution quadrature rule for highly oscillatory problems. With the help of operational calculus, the convergence rate of the convolution quadrature rule with respect to the frequency is derived. Furthermore, its application to highly oscillatory integral equations is also investigated. Numerical results are presented to verify the effectiveness of the convolution quadrature rule in solving highly oscillatory problems. It is found from theoretical and numerical results that the convolution quadrature rule for solving highly oscillatory problems is efficient and high-potential.
\end{abstract}

Keywords: highly oscillatory; convolution quadrature rule; volterra integral equation; Bessel kernel; convergence

\section{Introduction}

Highly oscillatory integrals (HOI) arise frequently in antenna problems involving Sommerfeld integrals (see [1,2]), computation of mutual impedance between conductors (see [3,4]) and many other oscillatory problems (HOP). Generally, an oscillatory integral can be written as

$$
I[f]=\int_{0}^{b} f(t) W(t) d t .
$$

Here $W(\cdot)$ denotes a highly oscillatory function and $f(\cdot)$ is slowly varied. Due to the high oscillation, classical quadrature rules (e.g., Newton-Cotes and Gauss rules ) are often ineffective, and calculation of this class of integrals is deemed to be a challenging problem ([5]).

Past decades witness a rapid development of researches on calculation of HOIs. Based on Filon's idea ([6]), Iserles and Nørsett developed the Filon-type method by approximating the slowly varied function by its Hermite interpolant. Both of theoretical and numerical results manifested that this method enjoyed high-order convergence rates with respect to the frequency ([7]). To get stable and fast algorithms, Domínguez, et al. ([8]), and Xiang, et al. ([9]), proposed the Clenshaw-Curtis-Filon-type method, respectively, which enjoyed extensive applications at present.

Although Filon's methodology leads to many efficient algorithms, most of them suffer to complicate computation of moment integrals. An alternative way to addressing this problem is transforming the integral interval into the complex plane, which derives the numerical steepest descent method. In [10], the complex integration method in the standard case was discussed extensively. In [11], the general form and corresponding error analysis were studied. This method was extended to the case of HOIs on semi-finite intervals in [12]. It is notable that the numerical steepest descent 
method with Gauss-Laguerre quadrature may not provide satisfactory solutions in practice (see [13]). Therefore, computation of transformed integrals is still an interesting topic.

Another important quadrature rule for HOIs is Levin's method ([14]). It enjoys a wide application for its being free of computing complex moments and less restrictions to integrands. The spirit of this method is transforming the integration problem into an ODE, and solving this equation by collocation methods. It is well-know that implementing of Levin methods comes down to efficient solutions of linear systems, which are often singular and dense. In [15], an SVD solver for the ill-conditioned system was presented. In [16], Olver developed a moment-free method by using the shifted GMRES. Recently, by employing the property of Chebyshev polynomials and preconditioners, a sparse and well-conditioned Levin method was constructed in [17].

There are many other important methods for calculating HOIs, for example, the homotopy perturbation method ([18]), the generalized quadrature rule ([19]), the extrapolation method ([20]). For simplicity, we omit the details. It is quite unexpected that little attention has been paid to the convolution quadrature rule (CQ) for HOIs ([21,22]), especially its asymptotic property in the case of high oscillation. In fact, CQ is well-known for its efficient in evaluating convolution integrals, and oscillatory integrals of convolution-type play significant roles in solving oscillatory and evolutionary problems (see $[23,24]$ ). Therefore, it is a meaningful issue to study CQ for HOIs.

Consider integral transforms with Bessel kernels as

$$
\int_{0}^{x} f(t) J_{m}(\omega(x-t)) d t, x \in[0, T]
$$

with $m \geq 0$ being an integer and $\omega \gg 1$, and oscillatory Volterra integral equations as

$$
\int_{0}^{x} J_{0}(\omega(x-t)) u(t) d t=f(x), x \in[0, T],
$$

where $f(\cdot)$ is sufficiently smooth, $f(0)=0$, and $u(t)$ is unknown. In this paper, we are devoted to studying convergence property of $C Q$ with respective to the frequency for solving above two problems. The same models have been considered in $[25,26]$, where authors concluded that Filon-type methods enjoyed the property that the higher the oscillation, the better the approximation. In the following, we will find CQ share a similar property as Filon-type methods, and even better when they are applied to solving highly oscillatory integral equations. The remaining parts are organized as follows. In Section 2, we briefly review CQ and give the convergence analysis. A modified rule is also proposed in this part. Then we study CQ for solving Volterra integral equations with highly oscillatory kernels in Section 3. Some numerical experiments are carried out in Section 4 to verify our given results.

\section{Convergence of the Convolution Quadrature Rule}

In this section, we revisit Lubich's convolution quadrature firstly. Then the convergence property with respect to the frequency is studied. In [21,22], Lubich proposed an algorithm for computing the following integral,

$$
I[f, g]=\int_{0}^{x} f(t) g(x-t) d t, x>0 .
$$

Let $F(\cdot)$ denote the Laplace transform of $f(\cdot)$ and satisfy

- $F(s)$ is analytic in the region $|\arg (s-c)|<\pi-\varphi, \varphi<\pi / 2, c \in \mathbb{R}$;

- there exist constants $M$ and $\mu$, such that $|F(s)| \leq M|s|^{-\mu}$.

By the definition of Laplace transform, it follows that

$$
f(t)=\frac{1}{2 \pi i} \int_{\Gamma} F(\lambda) e^{\lambda t} d \lambda
$$


where $\Gamma$ is a curve locates in the analytic region of $F(s)$ and goes from $\infty \cdot e^{-i(\pi-\varphi)}$ to $\infty \cdot e^{i(\pi-\varphi)}$.

Substituting (5) into (4) gives

$$
\int_{0}^{x} f(t) g(x-t) d t=\frac{1}{2 \pi i} \int_{\Gamma} F(\lambda) \int_{0}^{x} e^{\lambda t} g(x-t) d t d \lambda .
$$

Noting that $y(x)=\int_{0}^{x} e^{\lambda t} g(x-t) d t$ satisfies the initial value problem

$$
\left\{\begin{array}{l}
\frac{d y}{d x}=\lambda y(x)+g(x), \\
y(0)=0 .
\end{array}\right.
$$

Defining the grid $\left\{t_{n}:=n h, n=0,1, \ldots\right\}$, we can approximate $y(x)$ by

$$
\sum_{j=0}^{k} \alpha_{j} y_{n+j-k}=h \beta\left(\lambda y_{n}+g(n h)\right), n \geq 0,
$$

where $y_{-k}=\ldots=y_{-1}=0, g(x)=0(x<0)$. Multiplying both sides by $\zeta^{n}(n \geq 0)$ in (8) and summing give

$$
\left(\alpha_{0} \zeta^{k}+\ldots+\alpha_{k}\right) \mathbf{y}(\zeta)=\beta(h \lambda \mathbf{y}(\zeta)+h \mathbf{g}(\zeta))
$$

where $\mathbf{y}(\zeta)=\sum_{n=0}^{\infty} y_{n} \zeta^{n}, \mathbf{g}(\zeta)=\sum_{n=0}^{\infty} g(n h) \zeta^{n}$. Letting

$$
\delta(\zeta)=\frac{\alpha_{0} \zeta^{k}+\ldots+\alpha_{k}}{\beta}
$$

it follows that

$$
\mathbf{y}(\zeta)=\left(\frac{\delta(\zeta)}{h}-\lambda\right)^{-1} \mathbf{g}(\zeta)
$$

Since $F(s)$ is analytic in the inside region of the curve $\Gamma$, we have, by Cauchy's integral formula (see [27], p. 32),

$$
\frac{1}{2 \pi i} \int_{\Gamma} F(\lambda)\left(\frac{\delta(\zeta)}{h}-\lambda\right)^{-1} d \lambda=F\left(\frac{\delta(\zeta)}{h}\right) .
$$

Therefore, it follows that

$$
\begin{aligned}
\frac{1}{2 \pi i} \int_{\Gamma} F(\lambda) \mathbf{y}(\zeta) d \lambda & =\frac{1}{2 \pi i} \int_{\Gamma} F(\lambda)\left(\frac{\delta(\zeta)}{h}-\lambda\right)^{-1} \mathbf{g}(\zeta) d \lambda \\
& =F\left(\frac{\delta(\zeta)}{h}\right) \mathbf{g}(\zeta) .
\end{aligned}
$$

Here the coefficient corresponding to $\zeta^{n}$ denotes an approximation to the integral (4) at $x=n h$. Suppose

$$
F\left(\frac{\delta(\zeta)}{h}\right)=\sum_{j=0}^{\infty} w_{j}(h) \zeta^{j}
$$


Then CQ for (4) is defined as

$$
Q_{h}^{c q}(x)=\sum_{0 \leq j h \leq x} w_{j}(h) g(x-j h)
$$

By the definition of coefficients of Taylor expansions, we get

$$
w_{n}(h)=\frac{1}{2 \pi i} \int_{|z|=\rho} F\left(\frac{\delta(\zeta)}{h}\right) z^{-n-1} d z,
$$

where $\rho$ is sufficiently small such that the disc $|z| \leq \rho$ falls in the analytic region of $F(\delta(\zeta) / h)$. Letting $z=\rho e^{i \theta}$, we obtain

$$
w_{n}(h)=\frac{\rho^{-n}}{2 \pi} \int_{0}^{2 \pi} F\left(\frac{\delta\left(\rho e^{i \theta}\right)}{h}\right) e^{-i n \theta} d \theta
$$

Discretizing $\int_{0}^{2 \pi} F\left(\frac{\delta\left(\rho e^{i \theta}\right)}{h}\right) e^{-i n \theta} d \theta$ by the composite trapezoid rule gives

$$
\begin{aligned}
\int_{0}^{2 \pi} F\left(\frac{\delta\left(\rho e^{i \theta}\right)}{h}\right) e^{-i n \theta} d \theta & =\sum_{l=0}^{L-1} \int_{l \frac{2 \pi}{L}}^{(l+1) \frac{2 \pi}{L}} F\left(\frac{\delta\left(\rho e^{i \theta}\right)}{h}\right) e^{-i n \theta} d \theta \\
& \approx \sum_{l=0}^{L-1} \frac{\pi}{L}\left(F\left(\frac{\delta\left(\rho e^{i l \frac{2 \pi}{L}}\right)}{h}\right) e^{-i n l \frac{2 \pi}{L}}+F\left(\frac{\delta\left(\rho e^{i(l+1) \frac{2 \pi}{L}}\right)}{h}\right) e^{-i n(l+1) \frac{2 \pi}{L}}\right) \\
& =\frac{2 \pi}{L} \sum_{l=0}^{L-1} F\left(\frac{\delta\left(\rho e^{i l \frac{2 \pi}{L}}\right)}{h}\right) e^{-i n l \frac{2 \pi}{L}}
\end{aligned}
$$

The last equal sign works due to $F\left(\frac{\delta\left(\rho e^{i \theta}\right)}{h}\right) e^{-i n \theta}$ is $2 \pi$-periodic. This leads to

$$
w_{n}(h) \approx \frac{\rho^{-n}}{L} \sum_{l=0}^{L-1} F\left(\frac{\delta\left(\rho e^{i l \frac{2 \pi}{L}}\right)}{h}\right) e^{-i n l \frac{2 \pi}{L}}, n=0,1, \ldots, N
$$

Its computation complexity is $O(N \log N)$ by FFT. In this paper, we adopt $L=10 N$, $\rho^{N}=\sqrt{\epsilon}, \epsilon=10^{-16}$ to guarantee a precision of order $O(\sqrt{\epsilon})$ in (19).

Remark 1. In [28], convolution quadrature weights were rewritten as

$$
w_{j}(h)=\int_{0}^{\infty} g(s) \phi_{j}(s / h) d s .
$$

Here $\phi_{j}(t)=e^{-t} \frac{t^{j}}{j !}$ for backward differentiation formula of order 1 (BDF1), and $\phi_{j}(t)=$ $\frac{H_{j}(\sqrt{2 t})}{j !}\left(\frac{t}{2}\right)^{j / 2} e^{-3 t / 2}$ for BDF2 with $H_{j}(\cdot)$ denoting the $j$ th Hermite polynomial. Recurrence relations of frequently-used bases can be found in Table 1. 
Table 1. Recurrence relations for the CQ basis functions.

\begin{tabular}{|c|c|c|}
\hline Scheme & Initial Basis $\left(\phi_{-n}=0, n \geq 1\right)$ & Recurrence for Basis Functions $(j \geq 1)$ \\
\hline BDF1 & $\phi_{0}(t)=e^{-t}$ & $j \phi_{j}(t)-t \phi_{j-1}(t)=0$ \\
\hline BDF2 & $\phi_{0}(t)=e^{-3 t / 2}$ & $j \phi_{j}(t)-2 t \phi_{j-1}(t)+t \phi_{j-2}(t)=0$ \\
\hline BDF3 & $\phi_{0}(t)=e^{-11 t / 6}$ & $j \phi_{j}(t)-3 t \phi_{j-1}(t)+3 t \phi_{j-2}(t)-t \phi_{j-3}(t)=0$ \\
\hline BDF4 & $\phi_{0}(t)=e^{-25 t / 12}$ & $j \phi_{j}(t)-4 t \phi_{j-1}(t)+6 t \phi_{j-2}(t)-4 t \phi_{j-3}(t)+t \phi_{j-4}(t)=0$ \\
\hline
\end{tabular}

Existing convergence analysis of CQ often restricts to the property with respect to the stepsize. For example, setting

$$
\begin{aligned}
\mathcal{F}(s) g(x) & =\int_{0}^{x} f(t) g(x-t) d t, \\
\mathcal{F}\left(s_{h}\right) g(x) & =\sum_{0 \leq j h \leq x} w_{j}(h) g(x-j h),
\end{aligned}
$$

the convergence rate of $\mathrm{CQ}$ is as follows

Theorem 1. ([21], Theorem 3.1) Suppose that $\delta(\zeta)$ satisfies

- $\delta(\zeta)$ is analytic and without zeros in a neighbourhood of the closed unit disc $|\zeta| \leq 1$, with the exception of a zero at $\zeta=1$;

- $|\arg \delta(\zeta)| \leq \pi-\alpha$, for $|\zeta|<1$, for some $\alpha>\varphi$;

- $\quad \frac{\delta\left(e^{-h}\right)}{h}=1+O\left(h^{p}\right)$, for some $p \geq 1$.

Then we have

$$
\begin{aligned}
\left|\mathcal{F}\left(s_{h}\right) g(x)-\mathcal{F}(s) g(x)\right| \leq & C x^{\mu-1}\left\{h|g(0)|+\ldots+h^{p-1}\left|g^{(p-2)}(0)\right|\right. \\
& \left.+h^{p}\left(\left|g^{(p-1)}(0)\right|+x \max _{0 \leq t \leq x}\left|g^{(p)}(t)\right|\right)\right\}
\end{aligned}
$$

where the constant $C$ does not depend on the stepsize $h$.

One point which should be remarkable is that A-stable linear multistep methods for solving the initial value problem (7) are more reliable in actual computation. Therefore, in this paper, we make use of BDF2 in numerical experiments.

In the following parts, we study the convergence property of CQ for

$$
I[f]=\int_{0}^{b} f(t) J_{m}(\omega t) d t, \omega \gg 1 .
$$

Here $J_{m}(\cdot)$ denotes the first kind Bessel function of order $m$ with $m$ being a nonnegative integer. Defining $\tilde{f}(s):=f(b-s)$, we transform (1) into

$$
I[f]=\int_{0}^{b} \tilde{f}(b-s) J_{m}(\omega s) d s .
$$

Here the Laplace transform of $J_{m}(\omega s)$ can be represented as (see [29], p. 1024)

$$
F_{J_{m}}(s)=\frac{\left(\sqrt{s^{2}+\omega^{2}}-s\right)^{m}}{\omega^{m} \sqrt{s^{2}+\omega^{2}}} .
$$

Before elaborating on the convergence property of $C Q$, the following lemma should be presented first. 
Lemma 1. Suppose $f(\cdot)$ is continuous on the interval $S$, where $S$ may be a closed interval on the positive real axis or $[a, \infty)$ for some $a \geq 0$. Assume $\int_{S}|f(t)| d t, \int_{S}\left|f^{\prime}(t)\right| d t$ exist. Then for any $v>0$ and sufficiently large $\omega$, there exists a constant $C$, which does not depend on the frequency $\omega$, such that

$$
\left|\int_{S} f(t) J_{m}(\omega t) d t\right| \leq C \omega^{-1}
$$

Furthermore, if the interval $S$ does not contain origin, then it follows

$$
\left|\int_{S} f(t) J_{m}(\omega t) d t\right| \leq C \omega^{-3 / 2}
$$

This lemma can be proved by integration by parts and we omit the detail. Now the main theorem of this section follows.

Theorem 2. Suppose $f(\cdot) \in C^{2}[0, b]$, and $\int_{0}^{b}|f(t)| d t, \int_{0}^{b}\left|f^{\prime}(t)\right| d t, \int_{0}^{b}\left|f^{\prime \prime}(t)\right| d t$ exist. Then there exists a positive constant $C$ independent of $\omega$, such that, as the frequency $\omega$ tends to infinity, CQ satisfies

$$
\left|Q_{h}^{c q}(b)-I[f]\right| \leq C \omega^{-3 / 2} .
$$

Proof. Denote by $\delta_{j}$ the coefficients of $\delta(\zeta)=\sum_{j=0}^{\infty} \delta_{j} \tau^{j}$, and define

$$
s_{h} g(x):=\frac{1}{h} \sum_{0 \leq j h \leq x} \delta_{j} g(x-j h), 0<x \leq b .
$$

Then $I[f]$ can be represented as a Dunford-Taylor integral

$$
I[f]=\mathcal{F}\left(s_{h}\right) \tilde{f}(x)=\frac{1}{2 \pi i} \int_{\Gamma} F_{J_{m}}(\lambda)\left(s_{h}-\lambda\right)^{-1} \tilde{f}(x) d \lambda .
$$

By noting

$$
F_{J_{m}}(s)=\int_{0}^{\infty} e^{-t s} J_{m}(\omega t) d t
$$

we have

$$
\begin{aligned}
\mathcal{F}\left(s_{h}\right) \tilde{f}(x) & =\frac{1}{2 \pi i} \int_{\Gamma} \int_{0}^{\infty} e^{-t \lambda} J_{m}(\omega t)\left(s_{h}-\lambda\right)^{-1} \tilde{f}(x) d t d \lambda \\
& =\int_{0}^{\infty} J_{m}(\omega t) e^{-t s_{h}} \tilde{f}(x) d t
\end{aligned}
$$

This implies

$$
\begin{aligned}
\mathcal{F}\left(s_{h}\right) \tilde{f}(x)-\mathcal{F}(s) \tilde{f}(x)= & \int_{0}^{x} J_{m}(\omega t)\left(e^{-t s_{h}} \tilde{f}(x)-\tilde{f}(x-t)\right) d t \\
& +\int_{x}^{\infty} J_{m}(\omega t) e^{-t s_{h}} \tilde{f}(x) d t .
\end{aligned}
$$

Define $\phi_{1}(t):=e^{-t s_{h}} \tilde{f}(x)-\tilde{f}(x-t)$ and $\phi_{2}(t):=e^{-t s_{h}} \tilde{f}(x)$. By recurrence relations and derivatives of Bessel functions, we get 


$$
\begin{aligned}
\mathcal{F}\left(s_{h}\right) \tilde{f}(x)-\mathcal{F}(s) \tilde{f}(x)= & \phi_{1}(0) \int_{0}^{x} J_{m}(\omega t) d t+\left.\frac{1}{\omega}\left(\phi_{1}(t)-\phi_{1}(0)\right) J_{m+1}(\omega t)\right|_{t=0} ^{x} \\
& -\frac{1}{\omega} \int_{0}^{x}\left(\phi_{1}^{\prime}(t)-\frac{m+1}{t}\left(\phi_{1}(t)-\phi_{1}(0)\right)\right) J_{m+1}(\omega t) d t \\
& +\left.\frac{1}{\omega} \phi_{2}(t) J_{m+1}(\omega t)\right|_{t=x} ^{\infty} \\
& -\frac{1}{\omega} \int_{x}^{\infty}\left(\phi_{2}^{\prime}(t)-\frac{m+1}{t} \phi_{2}(t)\right) J_{m+1}(\omega t) d t .
\end{aligned}
$$

According to $\phi_{1}(0)=0$ and Lemma 1, it follows

$$
\left|\mathcal{F}\left(s_{h}\right) \tilde{f}(x)-\mathcal{F}(s) \tilde{f}(x)\right| \leq C \omega^{-3 / 2},
$$

where the constant $C$ does not depend on $\omega$. This completes the proof.

This theorem verifies the accuracy of CQ will increase as the frequency tends to infinity. According to the proof of Theorem 2, we can eliminate low order terms in (35) and get a modified convolution quadrature rule.

- $\quad$ Modified convolution quadrature rule for (1) of the first kind (MCQ1).

$$
Q_{h}^{m c q 1}(b)=\left.\mathcal{F}\left(s_{h}\right)(f(x)-f(b))\right|_{x=1}+f(b) \int_{0}^{b} J_{m}(\omega t) d t .
$$

According to ([30], p. 681), we have

$$
\int_{0}^{1} t^{\mu} J_{v}(\omega t) d t=\frac{2^{\mu} \Gamma\left(\frac{\mu+v+1}{2}\right)}{\omega^{\mu+1} \Gamma\left(\frac{v-\mu+1}{2}\right)}+\omega^{-\mu}(\mu+v-1) J_{v}(\omega) s_{\mu-1, v-1}^{(2)}(\omega)-\omega^{-\mu} J_{v-1}(\omega) s_{\mu, v}^{(2)}(\omega)
$$

Here $s_{\mu, \nu}^{(2)}(x)$ denotes the Lommel function of the second kind, and can be efficient computed by asymptotic expansions ([31]). This implies

$$
\int_{0}^{b} J_{m}(\omega t) d t=\frac{1}{b^{2} \omega}+\frac{(m-1) J_{m}(\omega b) s_{-1, m-1}^{(2)}(\omega b)-J_{m-1}(\omega b) s_{0, m}^{(2)}(\omega b)}{b} .
$$

Analogy to the proof of Theorem 2, we immediately arrive at the following results.

Theorem 3. Suppose $f(\cdot) \in C^{2}[0, b]$, and $\int_{0}^{b}|f(t)| d t, \int_{0}^{b}\left|f^{\prime}(t)\right| d t, \int_{0}^{b}\left|f^{\prime \prime}(t)\right| d t$ exist. Then there exists a positive constant $C$ independent of $\omega$, such that, as the frequency $\omega$ tends to infinity, MCQ1 satisfies

$$
\left|Q_{h}^{m c q 1}(b)-I[f]\right| \leq C \omega^{-2} .
$$

Remark 2. By Lubich's methodology of eliminating low order terms, we also obtain a modified convolution quadrature rule of the second kind for (1) (MCQ2). Although these two modified quadrature rules share the same convergence rates with respect to the stepsize, their convergence properties are quite different in the case of calculation of HOI. We will illustrate this phenomenon in Section 4. 


\section{Application to a Volterra Equation}

In literature, convolution quadrature rules are important tools for solving Volterra equations with convolution kernels ([32]). Many numerical experiments show they are efficient for solving some highly oscillatory Volterra integral equations (HOVIE). Consider HOVIE (3),

$$
\int_{0}^{x} J_{0}(\omega(x-t)) u(t) d t=f(x), x \in[0, T],
$$

where $f(\cdot)$ is sufficiently smooth and $f(0)=0$. Let

$$
I_{N}:=\left\{x_{n}: 0=x_{0}<x_{1}<\ldots<x_{N}=T\right\},
$$

be a uniform grid on $[0, T]$ with spacing $h:=T / N$. This equation arises from acoustic scattering problems (see [33]). By applying CQ to (3), we get

$$
\sum_{j=0}^{k} w_{k-j}(h) \tilde{u}_{j}=f_{k}, k=1,2, \ldots, N .
$$

Here $u_{j}$ denotes the numerical solution $\tilde{u}(x)$ at $x_{j}$ and $f_{k}:=f\left(x_{k}\right)$. Once the initial value $u_{0}$ is known, the numerical solution at the uniform grid $I_{N}$ can be obtained by solving the linear system (42), and the numerical solution on $[0, T]$ can be written as

$$
\tilde{u}(x):=\sum_{j=0}^{N} \tilde{u}_{j} \phi_{j}(x)
$$

Following the methodology from [24,26], we establish the convergence analysis by expressing the error function in terms of moments with highly oscillatory kernels. So let us consider some integrals involving Bessel kernels.

Lemma 2. Define the functional $T_{x}: C([0, T]) \rightarrow R$ as

$$
T_{x}(f)=\int_{0}^{x} J_{m}(\omega s) f(s) d s,
$$

with $x \in[0, T]$ and $f \in C([0, T])$. Then $\infty$-norm of the functional $T_{x}$ satisfies

$$
\left\|T_{x}\right\| \leq C \omega^{-1 / 2} \text {, as } \omega \rightarrow \infty,
$$

where $C$ is a constant independent of $\omega$.

Proof. It is easy to show

$$
\left\|T_{x}\right\|=\int_{0}^{x}\left|J_{m}(\omega s)\right| d s .
$$

By the variable transformation $t=\omega s$, we have

$$
\left\|T_{x}\right\|=\frac{1}{\omega} \int_{0}^{\omega x}\left|J_{m}(t)\right| d t .
$$


According to the asymptotic expansions of Bessel functions (see [34], p. 228), there exists $Z>0$ such that for any $z>Z$, and a constant $C^{*}$, we have

$$
\left|J_{m}(z)\right| \leq \frac{C^{*}}{\sqrt{z}} .
$$

This implies

$$
\begin{aligned}
\left\|T_{x}\right\| & =\frac{1}{\omega} \int_{0}^{Z}\left|J_{m}(t)\right| d t+\frac{1}{\omega} \int_{Z}^{\omega x}\left|J_{m}(t)\right| d t \\
& \leq \frac{1}{\omega} \int_{0}^{Z}\left|J_{m}(t)\right| d t+\frac{1}{\omega} \int_{Z}^{\omega x} \frac{C^{*}}{\sqrt{z}} d t .
\end{aligned}
$$

Therefore, there exists a constant $C$, such that

$$
\left\|T_{x}\right\| \leq C \omega^{-1 / 2}, \text { as } \omega \rightarrow \infty .
$$

This completes the proof.

Lemma 3. For any integers $\mu, v>0$ and $x \in(0, T]$, the following integral

$$
I=\omega^{1 / 2} \int_{0}^{x} \frac{J_{\mu}(\omega t) J_{v}(\omega(x-t))}{t} d t
$$

is uniformly bounded with respect to $\omega>0$.

Proof. The variable transformation $s=\omega t$ gives

$$
I=\omega^{1 / 2} \int_{0}^{\omega x} \frac{J_{\mu}(s) J_{v}(\omega x-s)}{s} d s .
$$

According to ([34], p. 242), we have

$$
I=\frac{\omega^{1 / 2} J_{\mu+v}(\omega x)}{\mu} .
$$

By the asymptotic expansion of Bessel functions, we completes the proof.

Now we get the convergence property of CQ for the numerical solution to HOVIE.

Theorem 4. Suppose that $f \in C^{3}[0, T]$, then the convolution quadrature rule for solving (3) introduces a unique numerical solution $\tilde{u}$, and satisfies

$$
|u(x)-\tilde{u}(x)| \leq C \omega^{-1 / 2}, x \in I_{N}, \omega \rightarrow \infty,
$$

where $C$ is a constant independent of $\omega$.

Proof. By noting

$$
\mathcal{F}\left(s_{h}\right) \tilde{u}(x)=f(x)
$$

and

$$
\mathcal{F}(s) u(x)=f(x)
$$


with $x \in I_{h}$, we get the error equation

$$
\mathcal{F}\left(s_{h}\right) \epsilon(x)-\left(\mathcal{F}(s) u(x)-\mathcal{F}\left(s_{h}\right) u(x)\right)=0,
$$

where $\epsilon(x):=\tilde{u}(x)-u(x)$. By Remark 1 and Lemma 1 , we have

$$
w_{j}(h)=\left\{\begin{array}{l}
O\left(\omega^{-1}\right), j=0 \\
O\left(\omega^{-3 / 2}\right), j>0
\end{array}\right.
$$

Therefore, the remaining work is proving $\mathcal{F}(s) u(x)-\mathcal{F}\left(s_{h}\right) u(x)$ behaves as $O\left(\omega^{-3 / 2}\right)$. A similar process to the proof of Theorem 2 gives

$$
\begin{aligned}
\mathcal{F}\left(s_{h}\right) u(x)-\mathcal{F}(s) u(x)= & \phi_{1}(0) \int_{0}^{x} J_{0}(\omega t) d t+\left.\frac{1}{\omega}\left(\phi_{1}(t)-\phi_{1}(0)\right) J_{1}(\omega t)\right|_{0} ^{x} \\
& -\frac{1}{\omega} \int_{0}^{x}\left(\phi_{1}^{\prime}(t)-\frac{1}{t}\left(\phi_{1}(t)-\phi_{1}(0)\right)\right) J_{1}(\omega t) d t \\
& +\left.\frac{1}{\omega} \phi_{2}(t) J_{1}(\omega t)\right|_{x} ^{\infty}-\frac{1}{\omega} \int_{x}^{\infty}\left(\phi_{2}^{\prime}(t)-\frac{1}{t} \phi_{2}(t)\right) J_{1}(\omega t) d t
\end{aligned}
$$

where $\phi_{1}(t):=e^{-t s_{h}} u(x)-u(x-t)$ and $\phi_{2}(t):=e^{-t s_{h}} u(x)$. Consider the integrals

$$
\tilde{I}:=\int_{0}^{x}\left(\phi_{1}^{\prime}(t)-\frac{1}{t}\left(\phi_{1}(t)-\phi_{1}(0)\right)\right) J_{1}(\omega t) d t+\int_{x}^{\infty}\left(\phi_{2}^{\prime}(t)-\frac{1}{t} \phi_{2}(t)\right) J_{1}(\omega t) d t .
$$

By using integration by parts we have

$$
\begin{aligned}
\tilde{I}= & \left.\frac{1}{\omega}\left(\phi_{1}^{\prime}(t)-\frac{\phi_{1}(t)}{t}\right) J_{2}(\omega t)\right|_{0} ^{x}-\frac{1}{\omega} \int_{0}^{x} \frac{t^{2} \phi_{1}^{\prime \prime}(t)-3 t \phi_{1}^{\prime}(t)+3 \phi_{1}(t)}{t^{2}} J_{2}(\omega t) d t \\
& +\left.\frac{1}{\omega}\left(\phi_{2}^{\prime}(t)-\frac{\phi_{2}(t)}{t}\right) J_{2}(\omega t)\right|_{x} ^{\infty}-\frac{1}{\omega} \int_{x}^{\infty}\left(\phi_{2}^{\prime \prime}(t)-\frac{3 \phi_{2}^{\prime}(t)}{t}+\frac{3 \phi_{2}(t)}{t^{2}}\right) J_{2}(\omega t) d t .
\end{aligned}
$$

According to [35], the exact solution to (3) can be written as

$$
u(x)=f^{\prime}(x)+\omega^{2} \int_{0}^{x} J_{0}(\omega s) f(x-s) d s-\omega \int_{0}^{x} J_{1}(\omega s) f^{\prime}(x-s) d s .
$$

Furthermore, we have

$$
\begin{aligned}
& u^{\prime}(x)=f^{\prime \prime}(x)+\omega^{2} \int_{0}^{x} J_{0}(\omega s) f^{\prime}(x-s) d s-\omega J_{1}(\omega x) f^{\prime}(0)-\omega \int_{0}^{x} J_{1}(\omega s) f^{\prime \prime}(x-s) d s, \\
& u^{\prime \prime}(x)=f^{\prime \prime \prime}(x)+\frac{\omega J_{1}(\omega x)}{x} f^{\prime}(0)+\omega \int_{0}^{x} \frac{J_{1}(\omega s)}{s} f^{\prime \prime}(x-s) d s .
\end{aligned}
$$

A direct calculation implies $\frac{t^{2} \phi_{1}^{\prime \prime}(t)-3 t \phi_{1}^{\prime}(t)+3 \phi_{1}(t)}{t^{2}}+\frac{\omega J_{1}(\omega(x-t))}{x-t} f^{\prime}(0)$ is $O(\omega)$ as $\omega$ goes to infinity. By Lemmas 2 and 3, we obtain

$$
\left|\int_{0}^{x} \frac{t^{2} \phi_{1}^{\prime \prime}(t)-3 t \phi_{1}^{\prime}(t)+3 \phi_{1}(t)}{t^{2}} J_{2}(\omega t) d t\right| \sim O\left(\omega^{1 / 2}\right), \omega \rightarrow \infty .
$$

With the help of Lemma 1, we have $\tilde{I}=O\left(\omega^{-1 / 2}\right)$. It follows that

$$
\left|\mathcal{F}(s) u(x)-\mathcal{F}\left(s_{h}\right) u(x)\right|=O\left(\omega^{-3 / 2}\right) .
$$


Combining Equations (58) and (66) gives

$$
|u(x)-\tilde{u}(x)| \leq C \omega^{-1 / 2}, x \in I, \omega \rightarrow \infty,
$$

where $C$ is a constant independent of $\omega$. This completes the proof.

\section{Numerical Results}

In this section, we present some numerical results to verify given estimates in previous sections. All experiments are performed in MATLAB 2013b.

As a first example, we consider the following HOI,

$$
I_{1}=\int_{0}^{1} J_{0}(\omega t) \frac{1}{1+25 t^{2}} d t, I_{2}=\int_{0}^{2} J_{1}(\omega t) \cos (t) \mathrm{e}^{-t} d t .
$$

In Figures 1-3, we show the convergence rates of three convolution quadrature rules. Slowly varied lines in these figures manifest that given asymptotic orders in Section 2 are optimal. Absolute errors of these methods are given in Tables 2 and 3. The numerical results illustrate MCQ1 is much more efficient than other two methods in computing HOI.

Table 2. Comparisons of quadrature rules for $\int_{0}^{1} J_{0}(\omega t) \frac{1}{1+25 t^{2}} d t$.

\begin{tabular}{cccccccc}
\hline $\boldsymbol{\omega}$ & $\mathbf{2 0}$ & $\mathbf{1 0 0}$ & $\mathbf{2 0 0}$ & $\mathbf{4 0 0}$ & $\mathbf{6 0 0}$ & $\mathbf{8 0 0}$ & $\mathbf{1 0 0 0}$ \\
\hline CQ & $9.7 \times 10^{-4}$ & $3.4 \times 10^{-5}$ & $1.1 \times 10^{-5}$ & $9.4 \times 10^{-7}$ & $1.5 \times 10^{-6}$ & $1.3 \times 10^{-6}$ & $1.7 \times 10^{-7}$ \\
MCQ1 & $8.3 \times 10^{-4}$ & $5.0 \times 10^{-6}$ & $6.3 \times 10^{-7}$ & $6.6 \times 10^{-8}$ & $2.1 \times 10^{-8}$ & $1.2 \times 10^{-8}$ & $7.2 \times 10^{-9}$ \\
MCQ2 & $9.4 \times 10^{-4}$ & $3.5 \times 10^{-5}$ & $1.1 \times 10^{-5}$ & $9.4 \times 10^{-7}$ & $1.5 \times 10^{-6}$ & $1.3 \times 10^{-6}$ & $1.7 \times 10^{-7}$ \\
\hline
\end{tabular}

Table 3. Comparisons of quadrature rules for $\int_{0}^{2} J_{1}(\omega t) \cos (t) \mathrm{e}^{-t} d t$.

\begin{tabular}{cccccccc}
\hline $\boldsymbol{\omega}$ & $\mathbf{2 0}$ & $\mathbf{1 0 0}$ & $\mathbf{2 0 0}$ & $\mathbf{4 0 0}$ & $\mathbf{6 0 0}$ & $\mathbf{8 0 0}$ & $\mathbf{1 0 0 0}$ \\
\hline CQ & $1.3 \times 10^{-5}$ & $7.8 \times 10^{-6}$ & $1.1 \times 10^{-5}$ & $1.3 \times 10^{-6}$ & $1.4 \times 10^{-6}$ & $1.4 \times 10^{-6}$ & $4.0 \times 10^{-7}$ \\
MCQ1 & $6.3 \times 10^{-6}$ & $9.3 \times 10^{-7}$ & $1.6 \times 10^{-7}$ & $2.5 \times 10^{-8}$ & $1.9 \times 10^{-8}$ & $9.2 \times 10^{-9}$ & $4.6 \times 10^{-9}$ \\
MCQ2 & $1.3 \times 10^{-5}$ & $7.8 \times 10^{-6}$ & $1.1 \times 10^{-5}$ & $1.3 \times 10^{-6}$ & $1.4 \times 10^{-6}$ & $1.4 \times 10^{-6}$ & $4.0 \times 10^{-7}$ \\
\hline
\end{tabular}
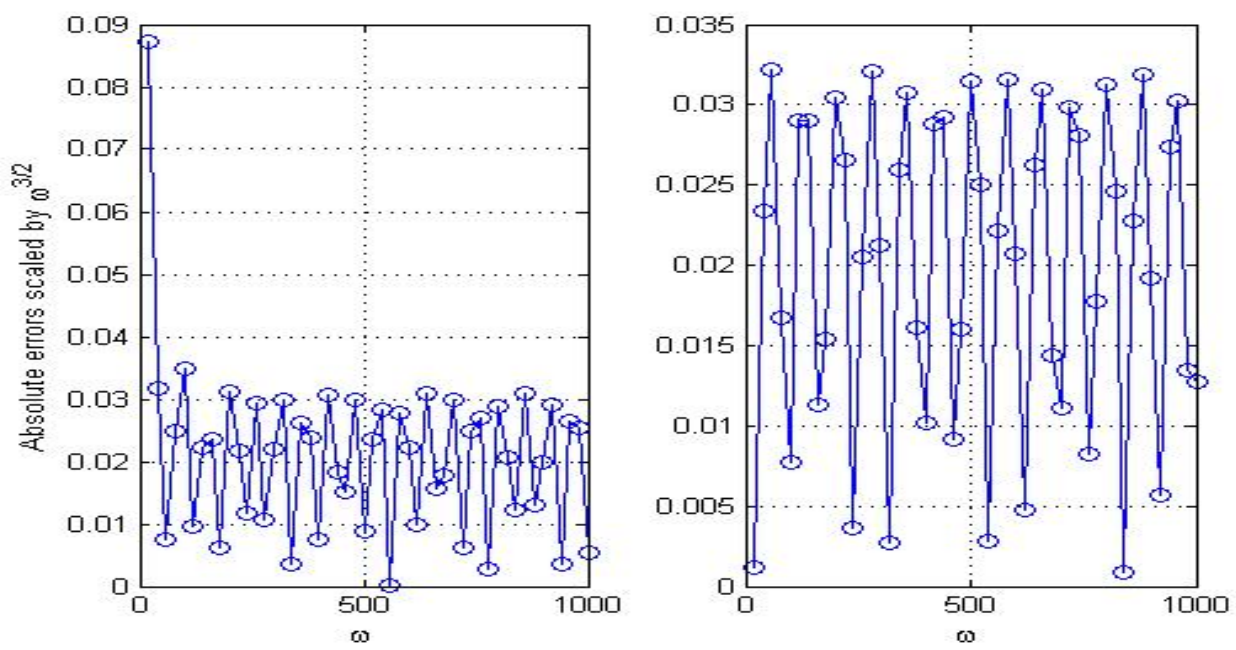

Figure 1. Asymptotic convergence rates of CQ for $\int_{0}^{1} J_{0}(\omega t) \frac{1}{1+25 t^{2}} d t$ (left) and $\int_{0}^{2} J_{1}(\omega t) \cos (t) \mathrm{e}^{-t} d t$ (right). 

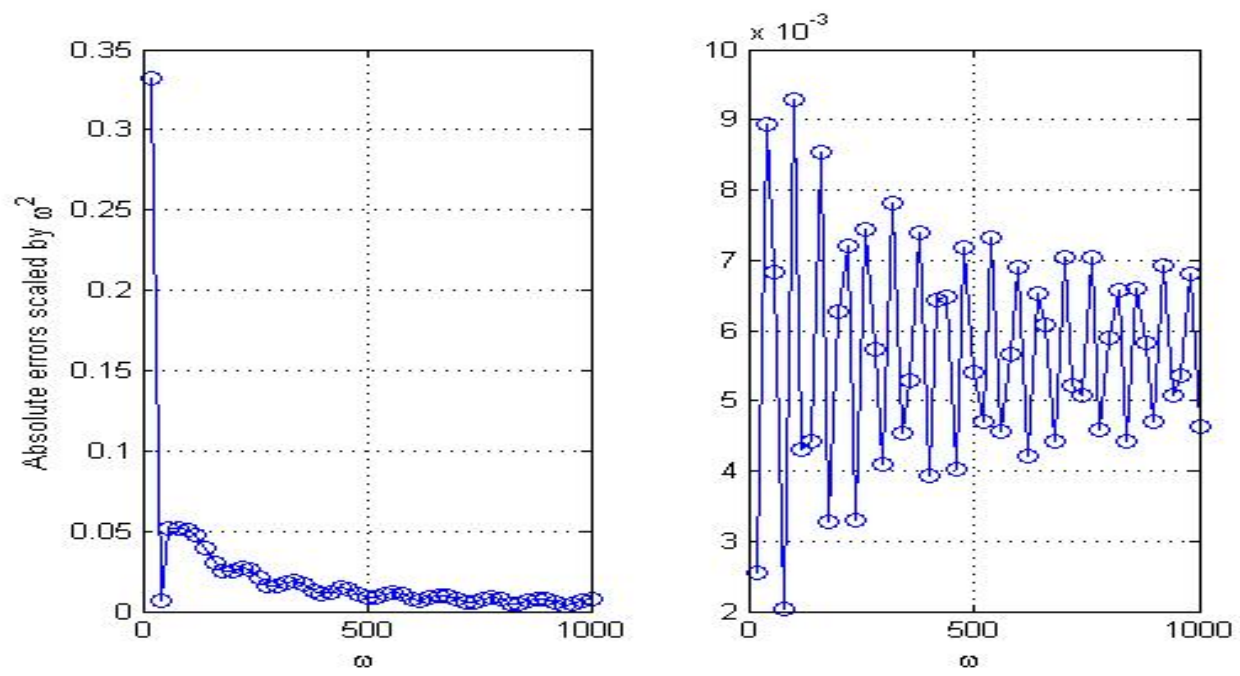

Figure 2. Asymptotic convergence rates of MCQ1 for $\int_{0}^{1} J_{0}(\omega t) \frac{1}{1+25 t^{2}} d t$ (left) and $\int_{0}^{2} J_{1}(\omega t) \cos (t) \mathrm{e}^{-t} d t$ (right).
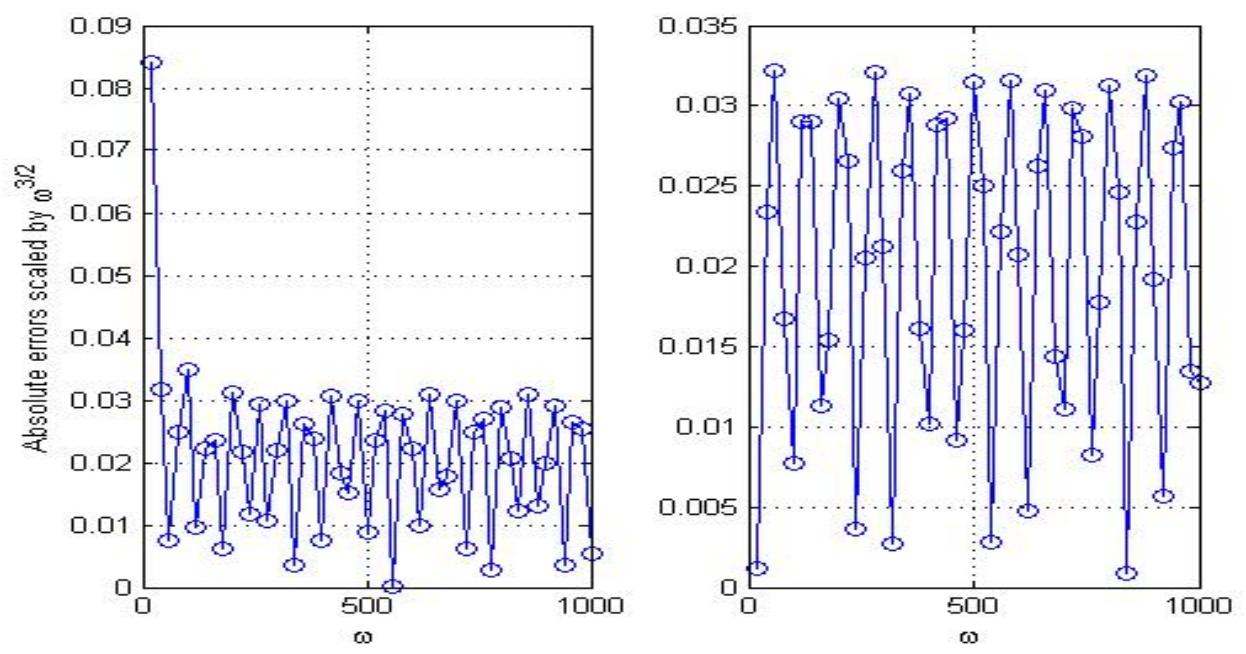

Figure 3. Asymptotic convergence rates of MCQ2 for $\int_{0}^{1} J_{0}(\omega t) \frac{1}{1+25 t^{2}} d t$ (left) and $\int_{0}^{2} J_{1}(\omega t) \cos (t) \mathrm{e}^{-t} d t$ (right)

In the second example, we consider application of CQ to solving HOVIE (3). Firstly, by letting $T=2$ and $N=20$, we give the computed solution in Table 4 with various $\omega$. Absolute errors listed in this table show CQ shares the same property as Filon methods $([24,26])$, that is, the higher the oscillation, the better the approximation. Then we compare these two methods in Figure 4 , where we can learn CQ behaves better than Filon methods. 
Table 4. CQ for Volterra integral equations with $f(x)=x \mathrm{e}^{-x}$.

\begin{tabular}{cccccccc}
\hline $\boldsymbol{\omega}$ & $\boldsymbol{x}=\mathbf{0 . 1}$ & $\boldsymbol{x}=\mathbf{0 . 4}$ & $\boldsymbol{x}=\mathbf{0 . 8}$ & $\boldsymbol{x}=\mathbf{1 . 2}$ & $\boldsymbol{x}=\mathbf{1 . 6}$ & $\boldsymbol{x}=\mathbf{1 . 8}$ & $\boldsymbol{x}=\mathbf{2}$ \\
\hline 10 & $3.3 \times 10^{-1}$ & $7.6 \times 10^{-2}$ & $4.7 \times 10^{-2}$ & $2.5 \times 10^{-2}$ & $8.0 \times 10^{-3}$ & $1.4 \times 10^{-2}$ & $3.1 \times 10^{-3}$ \\
100 & $9.1 \times 10^{-2}$ & $5.5 \times 10^{-3}$ & $6.3 \times 10^{-4}$ & $7.0 \times 10^{-5}$ & $3.5 \times 10^{-4}$ & $3.6 \times 10^{-5}$ & $2.6 \times 10^{-4}$ \\
200 & $5.0 \times 10^{-2}$ & $1.4 \times 10^{-4}$ & $3.6 \times 10^{-4}$ & $1.1 \times 10^{-4}$ & $1.2 \times 10^{-4}$ & $1.1 \times 10^{-4}$ & $1.8 \times 10^{-5}$ \\
500 & $1.9 \times 10^{-2}$ & $1.9 \times 10^{-4}$ & $1.2 \times 10^{-5}$ & $4.6 \times 10^{-5}$ & $3.7 \times 10^{-5}$ & $2.2 \times 10^{-5}$ & $6.8 \times 10^{-6}$ \\
1000 & $1.0 \times 10^{-2}$ & $5.9 \times 10^{-7}$ & $3.9 \times 10^{-5}$ & $1.2 \times 10^{-5}$ & $8.7 \times 10^{-10}$ & $9.6 \times 10^{-6}$ & $9.2 \times 10^{-6}$ \\
\hline
\end{tabular}
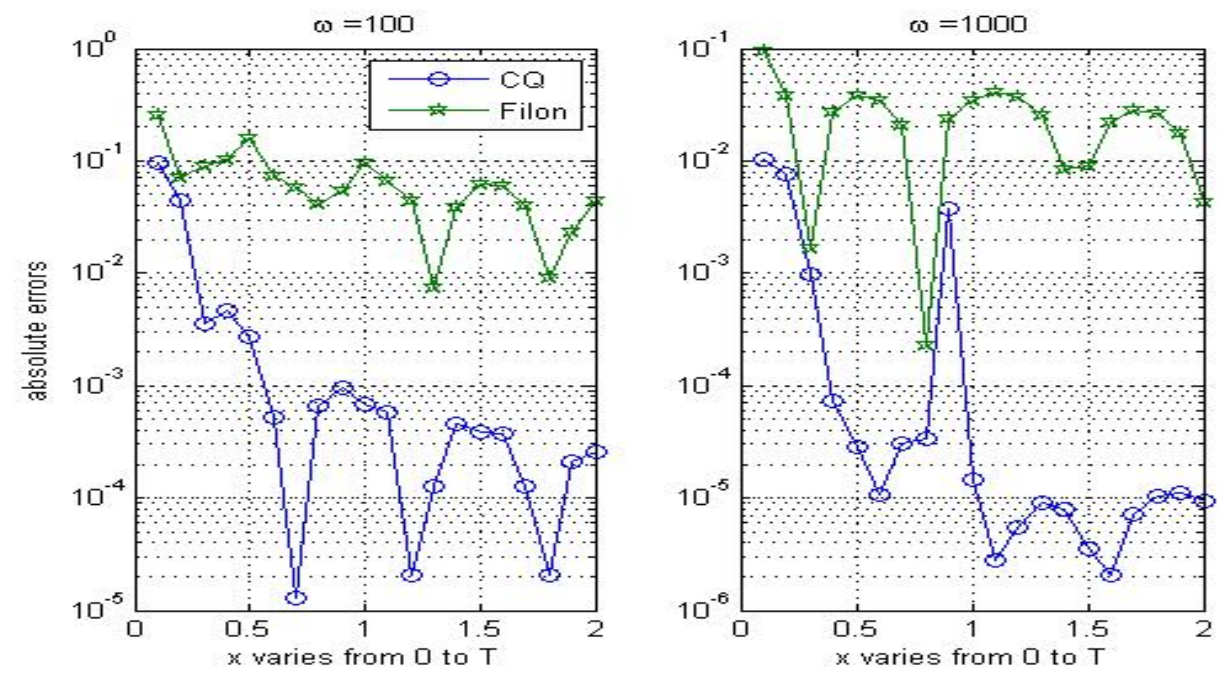

Figure 4. Comparisons between Filon methods and $\mathrm{CQ}$ for solving Volterra equations with $f(x)=\frac{x}{1+x^{2}}$.

\section{Conclusions}

The above theoretical and numerical results contribute to the study on the convergence property of CQ for solving HOP. The theoretical results in Section 2 reveal the convergence rate of CQ with respect to the frequency, that is, CQ converges in negative powers of $\omega$ as $\omega$ goes to infinity. Among them, the new modified rule (MCQ1) enjoys the fastest convergence rate. When we apply CQ to solving HOVIE, similar phenomenon is detected and analyzed. The numerical results in Section 4 show given convergence orders in Section 2 are optimal. In addition, this paper merely opens a window to the convergence theory of $\mathrm{CQ}$ for $\mathrm{HOP}$, much work on various versions of CQ, such as Runge-Kutta CQ ([36]), Fourier CQ ([37]), and so forth, is needed in the future.

Author Contributions: J.M. and H.L. conceived and designed the experiments; J.M. performed the experiments; H.L. analyzed the data; J.M. contributed reagents/materials/analysis tools; J.M. and H.L. wrote the paper.

Funding: This work is supported by NSF of China (No. 11761020), Scientific Research Foundation for Young Talents of Department of Education of Guizhou Province (No. 2016125), and Science and Technology Foundation of Guizhou Province (No. QKH[2017]5788).

Acknowledgments: The authors thank referees for their helpful suggestions.

Conflicts of Interest: The authors declare no conflict of interest. 


\section{Abbreviations}

The following abbreviations are used in this manuscript:

BDF backward differentiation formula

CQ convolution quadrature rule

FFT fast Fourier transform

GMRES generalized minimal residual method

HOI highly oscillatory integral

HOP highly oscillatory problem

ODE ordinary differential equation

HOVIE highly oscillatory Volterra integral equation

MCQ1 modified convolution quadrature of the first kind

MCQ2 modified convolution quadrature of the second kind

\section{References}

1. Michalski, K. Extrapolation methods for sommerfeld integral tails. IEEE Trans. Antennas Propag. 1998, 46, 1405-1418. [CrossRef]

2. Rakov, V.; Uman, M. Review and evaluation of lightning return stroke models including some aspects of their application. IEEE Trans. Electromagn. C 1998, 40, 403-426. [CrossRef]

3. Theodoulidis, T. Exact solution of Pollaczek's integral for evaluation of earth-return impedane for underground conductors. IEEE Trans. Electromagn. C 2012, 54, 806-814. [CrossRef]

4. Ma, J. Fast and high-precision calculation of earth return mutual impedance between conductors over a multilayered soil. COMPEL 2018, 37, 1214-1227. [CrossRef]

5. Iserles, A. On the numerical quadrature of highly-oscillating integrals I: Fourier transforms. IMA J. Numer. Anal. 2004, 24, 365-391. [CrossRef]

6. Filon, L.N.G. On a quadrature formula for trigonometric integrals. Proc. R. Soc. Edinb. 1928, $49,38-47$. [CrossRef]

7. Iserles, A.; Nørsett, S.P. Efficient quadrature of highly oscillatory integrals using derivatives. Proc. R. Soc. A 2005, 461, 1383-1399. [CrossRef]

8. Domínguez, V.; Graham, I.G.; Smyshlyaev, V.P. Stability and error estimates for Filon-Clenshaw-Curtis rules for highly-oscillatory integrals. IMA J. Numer. Anal. 2011, 31, 1253-1280. [CrossRef]

9. Xiang, S.; Cho, Y.; Wang, H.; Brunner, H. Clenshaw-Curtis-Filon-type methods for highly oscillatory Bessel transforms and applications. IMA J. Numer. Anal. 2011, 31, 1281-1314. [CrossRef]

10. Milovanovíc, G. Numerical calculation of integrals involving oscillatory and singular kernels and some applications of quadratures. Comput. Math. Appl. 1998, 36, 19-39. [CrossRef]

11. Huybrechs, D.; Vandewalle, S. On the evaluation of highly oscillatory integrals by analytic continuation. SIAM J. Numer. Anal. 2006, 44, 1026-1048. [CrossRef]

12. Majidian, H. Numerical approximation of highly oscillatory integrals on semi-finite intervals by steepest descent method. Numer. Algorithms 2013, 63, 537-548. [CrossRef]

13. Ma, J. Implementing the complex integral method with the transformed Clenshaw-Curtis quadrature. Appl. Math. Comput. 2015, 250, 792-797. [CrossRef]

14. Levin, D. Procedures for computing one- and two-dimensional integrals of functions with rapid irregular oscillations. Math. Comput. 1982, 38, 531-538. [CrossRef]

15. Li, J.; Wang, X.; Wang, T. A universal solution to one-dimensional highly oscillatory integrals. Sci. China 2008, 10, 1614-1622.

16. Olver, S. Shifted GMRES for oscillatory integrals. Numer. Math. 2010, 114, 607-628. [CrossRef]

17. Ma, J.; Liu, H. A well-conditioned Levin method for calculation of highly oscillatory integrals and its application. J. Comput. Appl. Math. 2018, 342, 451-462. [CrossRef]

18. Chen, R.; Xiang, S. Note on the homotopy perturbation method for multivariate vector-value oscillatory integrals. Appl. Math. Comput. 2009, 215, 78-84. [CrossRef]

19. Evans, G.A.; Chung, K.C. Some theoretical aspects of generalised quadrature rules. J. Complex. 2003, 19, 272-285. [CrossRef]

20. Sidi, A. Extrapolation method for oscillatory infinite integrals. Math. Comput. 1988, 51, 249-266. [CrossRef] 
21. Lubich, C. Convolution quadrature and discretized operational calculus. I. Numer. Math. 1988, 52, 129-145. [CrossRef]

22. Lubich, C. Convolution quadrature and discretized operational calculus. II. Numer. Math. 1988, 52, 413-425. [CrossRef]

23. Lubich, C. On convolution quadrature and Hille-Phillips operational calculus. Appl. Numer. Math. 1992, 9, 187-199. [CrossRef]

24. Xiang, S.; Brunner, H. Efficient methods for Volterra integral equations with highly oscillatory Bessel kernels. BIT Numer. Math. 2013, 53, 241-263. [CrossRef]

25. Xiang, S.; Wang, H. Fast integration of highly oscillatory integrals with exotic oscillators. Math. Comput. 2010, 79, 829-844. [CrossRef]

26. Ma, J.; Xiang, S.; Kang, H. On the convergence rates of Filon methods for the solution of a Volterra integral equation with a highly oscillatory Bessel kernel. Appl. Math. Lett. 2013, 26, 699-705. [CrossRef]

27. Stein, M.S.; Shakarchi, R. Complex Analysis; Princeton University Press: Princeton, NJ, USA, 2003.

28. Davis, P.; Duncan, D. Convolution spline approximations for time domain boundary integral equations. J. Integral Equ. Appl. 2014, 26, 369-410. [CrossRef]

29. Abramowitz, M.; Stegun, I.A. Handbook of Mathematical Functions; Dover Publications: Mineola, NY, USA, 1964.

30. Gradshteyn, I.S.; Ryzhik, I.M. Table of Integrals, Series, and Products; Academic Press: Cambridge, MA, USA, 1994.

31. Watson, G.N. A Treatise on the Theory of Bessel Functions; Cambridge University Press: Cambridge, UK, 1952.

32. Kauthen, J.P. A survey of singularly perturbed Volterra equations. Appl. Numer. Math. 1997, $24,95-114$. [CrossRef]

33. Davis, P.; Duncan, D. Stability and convergence of collocation schemes for retarded potential integral equations. SIAM J. Numer. Anal. 2004, 42, 1167-1188. [CrossRef]

34. Olver, F.J.; Lozier, D.W.; Boisvert, R.F.; Clark, C.W. NIST Handbook of Mathematical Functions; Cambridge University Press: Cambridge, UK, 2010.

35. Wang, H.; Xiang, S. Asymptotic expansion and Filon-type methods for a Volterra integral equation with a highly oscillatory kernel. IMA J. Numer. Anal. 2011, 31, 469-490. [CrossRef]

36. Banjai, L.; Lubich, C.; Melenk, J.M. Runge-Kutta convolution quadrature for operators arising in wave propagation. Numer. Math. 2011, 119, 1-20. [CrossRef]

37. Xu, K.; Austin, A.P.; Wei, K. A Fast Algorithm for the Convolution of Functions with Compact Support Using Fourier Extensions. SIAM J. Sci. Comput. 2017, 39, A3089-A3106. [CrossRef] 